Jeffreys' prior is the Hausdorff measure for the Hellinger and KullbackLeibler distances

Werner Doster

Fachbereich Mathematik, Universität Kaiserslautern

67663 Kaiserslautern, Germany

e-mail: doster@btbnet.de

September 24, 1998 


\begin{abstract}
On a family $\mathcal{F}:=\left\{P_{\vartheta} \mid \vartheta \in \Theta \subset \mathbb{R}^{k}\right\}$ of probability measures on a measure space $(\Omega, \mathcal{A})$ we consider the Hellinger and Kullback-Leibler distances. We show that under suitable regularity conditions Jeffreys' prior is proportional to the $k$-dimensional Hausdorff measure w.r.t. Hellinger distance respectively to the $\frac{k}{2}$-dimensional Hausdorff measure w.r.t. Kullback-Leibler distance. The proof is based on an area-formula for the Hausdorff measure w.r.t. to generalized distances.
\end{abstract}

Keywords: Hausdorff measure, Jeffreys' prior, area-formula, Hellinger distance, KullbackLeibler distance, Fisher Information

AMS classification numbers: 28A78, 


\title{
Jeffreys' prior is the Hausdorff measure for the Hellinger and Kullback-Leibler distances
}

\author{
Werner Doster \\ Fachbereich Mathematik, Universität Kaiserslautern \\ 67663 Kaiserslautern, Germany \\ e-mail: doster@btbnet.de \\ September 24, 1998
}

\section{Indroduction}

We consider a statistical experiment given by a family $\mathcal{F}:=\left\{P_{\vartheta} \mid \vartheta \in \Theta \subset \mathbb{R}^{k}\right\}$ of probability measures on a measure space $(\Omega, \mathcal{A})$ dominated by a $\sigma$-finite measure $\mu$ and we assume $P_{\vartheta} \neq P_{\vartheta^{\prime}}$ if $\vartheta \neq \vartheta^{\prime}$. By $\left\{f_{\vartheta} \mid \vartheta \in \Theta \subset \mathbb{R}^{k}\right\} \subset L^{1}(\mu)$ we denote the corresponding family of densities. On $\mathcal{F}$ there are several ways to measure the distance between two probability measures. In this paper, we consider the Hellinger metric (cf. [5, I.7.6]) $d\left(\vartheta, \vartheta^{\prime}\right):=\sqrt{\int_{\Omega}\left(\sqrt{f_{\vartheta}}-\sqrt{f_{\vartheta^{\prime}}}\right)^{2} d \mu}$ and the Kullback-Leibler distance (cf. [9]) $\mathcal{K}\left(\vartheta, \vartheta^{\prime}\right):=\int_{\Omega} f_{\vartheta} \ln \frac{f_{\vartheta}}{f_{\vartheta^{\prime}}} d \mu$.

The concept of Hausdorff measure gives a natural Borel regular outer measure on a metric space (cf. [12]). In our context this could be interpreted as a natural prior distribution on the parameter set $\Theta$. In [8, Theorem 7] and independently in [3, Theorem 2.3.2] an area-formula for Banach space valued functions is proved. So, if we assume $\Phi: \Theta \rightarrow L^{2}(\mu), \vartheta \mapsto \sqrt{f_{\vartheta}}$ to be locally Lipschitz-continuous the area-formula yields that $k$-dimensional Hausdorff measure w.r.t Hellinger distance is proportional to Jeffreys' prior (cf. [6, Section 3.10]). To prove the analogous result for Kullback-Leibler distance we first introduce the concept of Hausdorff measure w.r.t. generalized distances. Similar to the proof of the area-formula in [8] we get an area-formula for the Hausdorff measure w.r.t. such distances. Under suitable regularity conditions this gives that $\frac{k}{2}$-dimensional Hausdorff measure w.r.t. Kullback-Leibler distance is proportional to Jeffreys' prior (cf. Theorem 3.3).

\section{Hausdorff measures with respect to a generalized distance}

In this section we formulate some basic properties of the Hausdorff measure on a set $X$ w.r.t. maps $F: \mathbb{R}_{+} \rightarrow \mathbb{R}_{+}$and $f: X \times X \rightarrow \overline{\mathbb{R}}_{+}$. Most of the proofs in this section (cf. [3, Section 2.1]) can be given analogous to those of the corresponding propositions of the Hausdorff measure w.r.t. a metric (cf. [12, chap. I,II])

Let $X$ be a set, $F: \mathbb{R}_{+} \rightarrow \mathbb{R}_{+}$an increasing map and $f: X \times X \rightarrow \overline{\mathbb{R}}_{+}$a map. Then $\operatorname{diam}_{f}(A):=\sup \{f(x, y) \mid x, y \in A\}$ for sets $\emptyset \neq A \subset X$ and $\operatorname{diam}_{f}(\emptyset):=0$ defines the diameter of $A$ w.r.t. $f$.

For $\delta>0$ one can define outer measures on $X$ by

$\mathcal{H}_{\delta}^{F, f}(A):=\left\{\begin{array}{l}\inf \left\{\sum_{i=1}^{\infty} F\left(\operatorname{diam}_{f}\left(A_{i}\right)\right) \mid A \subset \cup_{i=1}^{\infty} A_{i}, \operatorname{diam}_{f}\left(A_{i}\right) \leq \delta, A_{i} \subset X\right\}, \text { if such a } \\ \text { covering }\left(A_{i}\right)_{i \in N} \text { exists } \\ \infty, \text { else }\end{array}\right.$

and

$$
\mathcal{H}^{F, f}(A):=\sup _{\delta>0} \mathcal{H}_{\delta}^{F, f}(A)
$$

(cf. [12, Theorem 15]).

Definition 2.1 The outer measure $\mathcal{H}^{F, f}$ on $X$ is called the Hausdorff measure on $X$ w.r.t. $(F, f)$. If $F(t):=t^{\alpha}$ for an $\alpha \geq 0$ we call $\mathcal{H}^{\alpha, f}:=\mathcal{H}^{F, f}$ the $\alpha$-dimensional Hausdorff measure on $X$ w.r.t. $f$.

If $\inf \{f(x, y) \mid x \in A, y \in E\}>0$, the sets $A, E \subset X$ are called $f$-separated. In the same way as $[12$, Theorem 16] the following lemma can be proved. 
Lemma 2.2 Let $X$ be a set, $f: X \times X \rightarrow \overline{\mathbb{R}}_{+}$a map with the property $f(x, y)=0$ iff $x=y$. Then $\mathcal{H}^{F, f}(A \cup E)=\mathcal{H}^{F, f}(A)+\mathcal{H}^{F, f}(E)$ for all $f$-separated sets $A, E \subset X$.

If $(X, d)$ is a metric space and $d$-separated sets are also $f$-separated, then $\mathcal{H}^{F, f}$ is a metric measure in the sense of [12, Definition 14] by lemma 2.2 and therefore by [12, Theorem 19] the Borel sets are $\mathcal{H}^{F, f}$-measurable. So we get the following corollary.

Corollary 2.3 Let $(X, d)$ be a metric space and $f: X \times X \rightarrow \overline{\mathbb{R}}_{+}$a map with the properties

(i) $f(x, y)=0$ iff $x=y$

(ii) d-separated sets are $f$-separated.

Then the Borel sets in $(X, d)$ are $\mathcal{H}^{F, f}$-measurable.

Remark 2.4 For strictly increasing continuous $F$ with $F(0)=0$ the Hausdorff measure w.r.t. $(F, f)$ coincides with the 1-dimensional Hausdorff measure w.r.t. $F \circ f$. In particular the $\alpha$ dimensional Hausdorff measure w.r.t. $f$ coincides with the 1-dimensional Hausdorff measure w.r.t. $f^{\alpha}$.

An important property of the Hausdorff measure is its behaviour under Hölder-continuous mappings. The following two lemmata can be proved analogously to the corresponding classical results (cf. [4, Prop. 2.2]).

Lemma 2.5 Let $X_{1}, X_{2}$ be sets and let $f_{i}: X_{i} \times X_{i} \rightarrow \overline{\mathbb{R}}_{+}, i=1,2$ and $\Phi: X_{1} \rightarrow X_{2}$ be mappings. Assume that there are constants $c \geq 0, \gamma \geq 0$ such that $f_{2}(\Phi(x), \Phi(y)) \leq c f_{1}(x, y)^{\gamma}$ for all $x, y \in X_{1}$. Then $\mathcal{H}^{\alpha, f_{2}}\left(\Phi\left(X_{1}\right)\right) \leq c^{\alpha} \mathcal{H}^{\alpha \gamma}, f_{1}\left(X_{1}\right)$.

If $\Phi$ is isometric, that is $f_{2}(\Phi(x), \Phi(y))=f_{1}(x, y)$ for all $x, y \in X_{1}$, then $\mathcal{H}^{\alpha, f_{2}}\left(\Phi\left(X_{1}\right)\right)=$ $\mathcal{H}^{\alpha, f_{1}}\left(X_{1}\right)$.

Lemma 2.6 Let $X_{1}, X_{2}$ be sets and let $f_{i}: X_{i} \times X_{i} \rightarrow \overline{\mathbb{R}}_{+}, i=1,2$ and $\Phi: X_{1} \rightarrow X_{2}$ be mappings. For each $x \in X_{1}$ we assume that there is a $\delta_{x}>0$ and a $L_{x}>0$ such that $f_{2}\left(\Phi\left(x^{\prime}\right), \Phi\left(x^{\prime \prime}\right)\right) \leq L_{x} f_{1}\left(x^{\prime}, x^{\prime \prime}\right)$ for all $x^{\prime}, x^{\prime \prime} \in\left\{y \in X_{1} \mid f_{1}(x, y) \leq \delta_{x}\right\}$. Then for $A \subset X_{1}$ one has $\mathcal{H}^{\alpha, f_{2}}(\Phi(A))=0$ if $\mathcal{H}^{\alpha, f_{1}}(A)=0$.

If $X$ is a vector space (over the field $\mathbb{R}$ ) and $\|\cdot\|$ is a seminorm on $X$ we denote by $B_{\|\cdot\|}$ the corresponding unit ball and by $\mathcal{H}^{k,\|\cdot\|}$ we denote the $k$-dimensional Hausdorff measure w.r.t. the pseudometric induced by the seminorm $\|\cdot\|$. If $\|\cdot\|$ is a norm on $\mathbb{R}^{k}$ (in this case we denote the norm by $|\cdot|$ ) the outer measure $\mathcal{H}^{k,|\cdot|}$ induces a translation invariant measure on the Borel sets, positive on each nonempty open set and finite on compact sets. By uniqueness of Haar measure on $\mathbb{R}^{k}$ and Borel regularity of both outer measures one concludes that $\mathcal{H}^{k,|\cdot|}$ is proportional to Lebesgue measure $\lambda^{k}$. The factor is given by $\frac{2^{k}}{\lambda^{k}\left(B_{|\cdot|}\right)}=\mathcal{H}^{k,|\cdot|}(W)$ (cf. [8, Lemma 6], [3, Korollar 2.2.3]) where $W:=\left\{x \in \mathbb{R}^{k} \mid 0 \leq x_{i} \leq 1\right.$ for all $\left.1 \leq i \leq k\right\}$ denotes the unit cube in $\mathbb{R}^{k}$. By $|\cdot|_{2}$ we denote Euclidean norm in Euclidean space $\mathbb{R}^{\bar{k}}$.

Similar to the arguments in the first part of the proof of [8, Theorem 7] we get an area-formula for the Hausdorff measure $\mathcal{H}^{k, f}$ on Borel sets $A \subset \mathbb{R}^{k}$.

Proposition 2.7 Let $A \subset \mathbb{R}^{k}$ be a Borel set and let $f: A \times A \rightarrow \bar{R}_{+}$be a map such that all Borel sets in $A$ are $\mathcal{H}^{k, f}$-measurable. Further we assume for each $x \in A$ that there is a norm $|\cdot|_{x}$ on $\mathbb{R}^{k}$ such that for each $\varepsilon>0$ there is a $\delta=\delta(x, \varepsilon)>0$ with

$$
(1-\varepsilon)\left|x^{\prime}-x^{\prime \prime}\right|_{x} \leq f\left(x^{\prime}, x^{\prime \prime}\right) \leq(1+\varepsilon)\left|x^{\prime}-x^{\prime \prime}\right|_{x}
$$

for all $x^{\prime}, x^{\prime \prime} \in B(x, \delta) \cap A=\left\{y \in A|| x-\left.y\right|_{2} \leq \delta\right\}$.

Then

$$
\mathcal{H}^{k, f}(E)=\int_{E} \frac{2^{k}}{\lambda^{k}\left(B_{|\cdot|_{x}}\right)} \lambda^{k}(d x)
$$

for all Borel sets $E \subset A$. 
Proof: Let $\varepsilon>0$ and $E \subset A$ be a Borel set. By Vitali's covering theorem we get a sequence $\left(x_{i}\right)_{i \in \mathbb{N}}$ in $E$ and $0<\delta_{i} \leq \delta\left(x_{i}, \varepsilon\right), \quad i \in \mathbb{N}$, such that the balls $B\left(x_{i}, \delta_{i}\right)$ are disjoint and $\lambda^{k}\left(E \backslash \bigcup_{i=1}^{\infty} B\left(x_{i}, \delta_{i}\right)\right)=0$. Moreover we have

$$
\frac{1-\varepsilon}{1+\varepsilon}\left|x^{\prime}-x^{\prime \prime}\right|_{x} \leq\left|x^{\prime}-x^{\prime \prime}\right|_{x_{i}} \leq \frac{1+\varepsilon}{1-\varepsilon}\left|x^{\prime}-x^{\prime \prime}\right|_{x}
$$

for all $x \in E \cap B\left(x_{i}, \delta_{i}\right), \quad x^{\prime}, x^{\prime \prime} \in E \cap B\left(x_{i}, \delta_{i}\right) \cap B(x, \delta(x, \varepsilon))$. Thus

$$
\begin{aligned}
& \mathcal{H}^{k,|\cdot|_{x_{i}}}(W) \lambda^{k}\left(E \cap B\left(x_{i}, \delta_{i}\right) \cap B(x, \delta(x, \varepsilon))\right)=\mathcal{H}^{k,|\cdot|_{x_{i}}}\left(E \cap B\left(x_{i}, \delta_{i}\right) \cap B(x, \delta(x, \varepsilon))\right) \\
& \quad \leq\left(\frac{1+\varepsilon}{1-\varepsilon}\right)^{k} \mathcal{H}^{k,|\cdot|_{x}}(W) \lambda^{k}\left(E \cap B\left(x_{i}, \delta_{i}\right) \cap B(x, \delta(x, \varepsilon))\right)
\end{aligned}
$$

and

$$
\begin{aligned}
& \mathcal{H}^{k,|\cdot|_{x_{i}}}(W) \lambda^{k}\left(E \cap B\left(x_{i}, \delta_{i}\right) \cap B(x, \delta(x, \varepsilon))\right) \\
& \quad \geq\left(\frac{1-\varepsilon}{1+\varepsilon}\right)^{k} \mathcal{H}^{k,|\cdot|_{x}}(W) \lambda^{k}\left(E \cap B\left(x_{i}, \delta_{i}\right) \cap B(x, \delta(x, \varepsilon))\right)
\end{aligned}
$$

for all $x \in E \cap B\left(x_{i}, \delta_{i}\right)$. Therefore we get

$$
\left(\frac{1-\varepsilon}{1+\varepsilon}\right)^{k} \mathcal{H}^{k,|\cdot|_{\boldsymbol{x}}}(W) \leq \mathcal{H}^{k,|\cdot|_{\boldsymbol{x}_{\boldsymbol{i}}}}(W) \leq\left(\frac{1+\varepsilon}{1-\varepsilon}\right)^{k} \mathcal{H}^{k,|\cdot|_{\boldsymbol{x}}}(W)
$$

for $\lambda^{k}$-almost all $x \in E \cap B\left(x_{i}, \delta_{i}\right)$. So using lemmata 2.2 up to 2.6 we have

$$
\begin{aligned}
\mathcal{H}^{k, f}(E) & =\mathcal{H}^{k, f}\left(\bigcup_{i=1}^{\infty}\left(E \cap B\left(x_{i}, \delta_{i}\right)\right)\right) \\
& =\sum_{i=1}^{\infty} \mathcal{H}^{k, f}\left(E \cap B\left(x_{i}, \delta_{i}\right)\right) \\
& \leq(1+\varepsilon)^{k} \sum_{i=1}^{\infty} \mathcal{H}^{k,|\cdot|_{x_{i}}}(W) \lambda^{k}\left(E \cap B\left(x_{i}, \delta_{i}\right)\right) \\
& \leq(1+\varepsilon)^{k} \sum_{i=1}^{\infty} \int_{E \cap B\left(x_{i}, \delta_{i}\right)} \mathcal{H}^{k,|\cdot|_{x_{i}}}(W) \lambda^{k}(d x) \\
& \leq \frac{(1+\varepsilon)^{2 k}}{(1-\varepsilon)^{k}} \sum_{i=1}^{\infty} \int_{E \cap B\left(x_{i}, \delta_{i}\right)} \mathcal{H}^{k,|\cdot|_{\boldsymbol{x}}}(W) \lambda^{k}(d x) \\
& =\frac{(1+\varepsilon)^{2 k}}{(1-\varepsilon)^{k}} \int_{E} \mathcal{H}^{k,|\cdot|_{\boldsymbol{x}}}(W) \lambda^{k}(d x)
\end{aligned}
$$

and analogously

$$
\frac{(1-\varepsilon)^{2 k}}{(1+\varepsilon)^{k}} \int_{E} \mathcal{H}^{k,|\cdot|_{x}}(W) \lambda^{k}(d x) \leq \mathcal{H}^{k, f}(E)
$$

With $\varepsilon$ declining to 0 this proves the lemma. 


\section{The area formula for Hausdorff measures with re- spect to Hellinger and Kullback-Leibler distances}

For this section let $\mathcal{F}:=\left\{P_{\vartheta} \mid \vartheta \in \Theta \subset \mathbb{R}^{k}\right\}$ be a family of probability measures on a measurable space $(\Omega, \mathcal{A})$ such that $P_{\vartheta} \neq P_{\vartheta^{\prime}}$ whenever $\vartheta \neq \vartheta^{\prime}$. We assume the family $\mathcal{F}$ to be dominated by a $\sigma$-finite measure $\mu$ and denote by $\left\{f_{\vartheta} \mid \vartheta \in \Theta\right\} \subset L^{1}(\mu)$ the corresponding density functions. If the map $\Phi: \Theta \rightarrow L^{2}(\mu), \vartheta \mapsto \sqrt{f_{\vartheta}}$ is differentiable at $\vartheta_{0} \in \Theta$ we call the matrix (w.r.t. the canonical algebraic basis) corresponding to the bilinear form $I\left(\vartheta_{0}\right):=4 D \Phi\left(\vartheta_{0}\right)^{*} D \Phi\left(\vartheta_{0}\right)$, the Fisher Information matrix of $\mathcal{F}$ at $\vartheta_{0} \in \Theta$ and in this case $|\cdot|_{\vartheta}$ denotes the seminorm on $\mathbb{R}^{k}$ induced by the Fisher Information matrix $I(\vartheta)$, i.e. $|y|_{\vartheta}:=\sqrt{\langle I(\vartheta) y, y\rangle}, y \in \mathbb{R}^{k}$.

On the set $\Theta \times \Theta$ we consider the Hellinger distance $d\left(\vartheta, \vartheta^{\prime}\right):=\left\|\Phi(\vartheta)-\Phi\left(\vartheta^{\prime}\right)\right\|_{2}$ (cf. [5, I.7.6]) and the Kullback-Leibler distance $\mathcal{K}\left(\vartheta, \vartheta^{\prime}\right):=\int_{\Omega} f_{\vartheta} \ln \frac{f_{\vartheta}}{f_{\vartheta^{\prime}}} d \mu$ (cf. [9]), respectively. In general for the Kullback-Leibler distance the triangle inequality failes so $\mathcal{K}$ is not a metric. One has $0 \leq \mathcal{K}\left(\vartheta, \vartheta^{\prime}\right) \leq \infty, \mathcal{K}\left(\vartheta, \vartheta^{\prime}\right)=0$ iff $\vartheta=\vartheta^{\prime}$ and $\mathcal{K}\left(\vartheta, \vartheta^{\prime}\right)<\infty$ implies that $P_{\vartheta}$ is absolutely continuous to $P_{\vartheta^{\prime}}$.

In the sense of section 1 one can consider the Hausdorff measure on $\Theta$ w.r.t. Hellinger distance and Kullback-Leibler distance, respectively.

Let us consider the following regularity conditions

C1: The parameter set $\Theta \subset \mathbb{R}^{k}$ is open and $\Phi: \Theta \longrightarrow L^{2}(\mu), \vartheta \mapsto \sqrt{f_{\vartheta}}$ is an injective and locally Lipschitz-continuous function.

C2: The parameter set $\Theta \subset \mathbb{R}^{k}$ is open and $\Phi$ is injective and twice continuously differentiable. Further $f_{\vartheta} \ln \frac{f_{\vartheta^{\prime}}}{f_{\vartheta}} \in L^{1}(\mu)$ for all $\vartheta, \vartheta^{\prime} \in \Theta$ and for each $\vartheta \in \Theta$ the map $\Psi_{\vartheta}: \Theta \longrightarrow L^{1}(\mu), \vartheta^{\prime} \mapsto f_{\vartheta} \ln \frac{f_{\vartheta}^{\prime}}{f_{\vartheta}}$ is twice continuously differentiable in $\vartheta^{\prime}$ and the second order derivative $D^{2} \Psi_{\vartheta}$ depends continuously on $\vartheta$ and $\vartheta^{\prime}$.

Remark: A real Banach space $E$ has the Radon-Nikdym Property (RNP) if every function $f:[0,1] \longrightarrow E$ of bounded variation is differentiable $\lambda^{1}$-a.e. Every reflexive Banach space has the (RNP). By Aronszajn's theorem (cf. [1, Lemma2, chapter II] and [11, Theorem 6]) every locally Lipschitz-continuous function $f: \mathbb{R}^{k} \supset A \rightarrow E, A$ open, is differentiable $\lambda^{k}$-a.e. Hence condition $\mathrm{C} 1$ implies $\Phi$ to be differentiable $\lambda^{k}$-a.e.

In the following we will show that under the regularity conditions $\mathrm{C} 1$ respectively $\mathrm{C} 2$ the $k$ dimensional Hausdorff measure w.r.t. Hellinger distance $d$ and the $\frac{k}{2}$-dimensional Hausdorff meausre w.r.t. Kullback-Leibler distance $\mathcal{K}$ are proportional to Jeffreys' prior on the Borel $\sigma$-algebra of $\Theta$, more precisely we have

$$
\begin{aligned}
\mathcal{H}^{k, d}(E \cap \Theta) & =\frac{1}{\omega_{k}} \int_{E \cap \Theta} \sqrt{\operatorname{det} I(\vartheta)} d \mu \quad \text { and } \\
\mathcal{H}^{\frac{k}{2}, \mathcal{K}}(E \cap \Theta) & =\frac{2^{\frac{k}{2}}}{\omega_{k}} \int_{E \cap \Theta} \sqrt{\operatorname{det} I(\vartheta)} d \mu
\end{aligned}
$$

for all Borel sets $E \subset \mathbb{R}^{k}$, where $\omega_{k}$ denotes the Lebesgue measure of the $k$-dimensional Euclidean unit ball and $I(\vartheta)$ denotes the Fisher Information matrix of the family $\left\{P_{\vartheta} \mid \vartheta \in \Theta\right\}$ at $\vartheta$.

The following proposition shows that under the regularity condition C2 the squared KullbackLeibler distance locally behaves as the seminorm $|\cdot|_{\vartheta}$ induced by the Fisher Information matrix.

Proposition 3.1 Let the regularity conditions $\mathrm{C} 2$ be fulfilled. Then for each $\vartheta_{0} \in \Theta$ and each $\varepsilon>0$ there is a $\delta>0$ such that

$$
\left|\vartheta-\vartheta^{\prime}\right|_{\vartheta_{0}}^{2}-\varepsilon\left|\vartheta-\vartheta^{\prime}\right|^{2} \leq 2 \mathcal{K}\left(\vartheta, \vartheta^{\prime}\right) \leq\left|\vartheta-\vartheta^{\prime}\right|_{\vartheta_{0}}^{2}+\varepsilon\left|\vartheta-\vartheta^{\prime}\right|^{2}
$$

for all $\vartheta, \vartheta^{\prime} \in B\left(\vartheta_{0}, \delta\right)$. 
Proof: Let $\vartheta, \vartheta^{\prime} \in \Theta$ and $x, y \in \mathbb{R}^{k}$. Let $\varphi: \Theta \rightarrow L^{1}(\mu), \vartheta \mapsto f_{\vartheta}$. Then $\varphi$ is differentiable with derivative $D \varphi(\vartheta)=2 \sqrt{f_{\vartheta}} D \Phi(\vartheta)$ at $\vartheta \in \Theta($ cf. [3, Satz 3.1.5], [13, Lemma 5.3]) and $\int D \varphi(\vartheta) \lambda^{k}(d \vartheta)=0=\int x^{t} D^{2} \varphi(\vartheta) y \lambda^{k}(d \vartheta)$. Using standard subsequence arguments (cf. [2, Theorem 20.8]) the differentiability assumptions yield that one can get the derivatives of $\Psi_{\vartheta}$ by formal differentation, i. e. $D \Psi_{\vartheta}\left(\vartheta^{\prime}\right) y=f_{\vartheta} \frac{D \varphi\left(\vartheta^{\prime}\right) y}{f_{\vartheta}}$ and $x^{t} D^{2} \Psi_{\vartheta}\left(\vartheta^{\prime}\right) y=f_{\vartheta} \frac{x^{t} D^{2} \varphi\left(\vartheta^{\prime}\right) y}{f_{\vartheta^{\prime}}}-$ $f_{\vartheta} \frac{x^{t} D \varphi\left(\vartheta^{\prime}\right)^{*} D \varphi\left(\vartheta^{\prime}\right) y}{f_{\vartheta^{\prime}}^{2}} \mu$-almost everywhere and Taylor expansion of $\Psi_{\vartheta}$ at the point $\vartheta$ yields $-\mathcal{K}(\vartheta, \vartheta+\Delta)=-\frac{1}{2}\langle\Delta, I(\vartheta) \Delta\rangle+\int r(\vartheta, \Delta) d \mu$, where $r(\vartheta, \Delta)$ denotes the error of the second order Taylor expansion of $\Psi_{\vartheta}$ at $\vartheta$.

Now let $\vartheta_{0} \in \Theta$ and $\varepsilon>0$. The Fisher Information matrix $I(\vartheta)$ depends continuously on $\vartheta$, because $\Phi$ is continuously differentiable. Hence there is a $\delta_{1}>0$ such that the closed ball $B\left(\vartheta_{0}, \delta_{1}\right)$ is contained in $\Theta$ and $\sup _{\vartheta \in B\left(\vartheta_{0}, \delta_{1}\right)}\left\|I\left(\vartheta_{0}\right)-I(\vartheta)\right\| \leq \frac{\varepsilon}{2}$. By the continuity of $\left(\vartheta, \vartheta^{\prime}\right) \mapsto D^{2} \Psi_{\vartheta}\left(\vartheta^{\prime}\right)$ there are $\delta_{2}>0, \delta_{3}>0$ such that $\sup _{\vartheta \in B\left(\vartheta_{0}, \delta_{3}\right)}\left|\int r(\vartheta, \Delta) d \mu\right| \leq \frac{\varepsilon}{4}|\Delta|_{2}^{2}$ for all $|\Delta|_{2} \leq \delta_{2}$. Take $\delta:=\frac{1}{2} \min \left\{\delta_{1}, \delta_{2}, \delta_{3}\right\}$, then we have $\left|\vartheta-\vartheta^{\prime}\right|_{2} \leq 2 \delta=\min \left\{\delta_{1}, \delta_{2}, \delta_{3}\right\}$ for all $\vartheta, \vartheta^{\prime} \in B\left(\vartheta_{0}, \delta\right)$. Therefore

$$
\begin{aligned}
\left|2 \mathcal{K}\left(\vartheta, \vartheta^{\prime}\right)-\left\langle\vartheta-\vartheta^{\prime}, I\left(\vartheta_{0}\right)\left(\vartheta-\vartheta^{\prime}\right)\right\rangle\right| & \leq \| I(\vartheta)-I\left(\vartheta_{0} \|\left|\vartheta-\vartheta^{\prime}\right|_{2}^{2}+2\left|\int r\left(\vartheta, \vartheta-\vartheta^{\prime}\right) d \mu\right|\right. \\
& \leq \varepsilon\left|\vartheta-\vartheta^{\prime}\right|^{2}
\end{aligned}
$$

This proves the proposition.

From the proof of theorem 3.3 below we separate the following lemma.

Lemma 3.2 Let $\|\cdot\|$ be a seminorm on $\mathbb{R}^{k}$ which is not a norm and let $M>0$ such that $\|x\| \leq M|x|_{2}$ for all $x \in \mathbb{R}^{k}$. For $\tau>0$ define $|x|_{\tau}:=\|x\|+\tau|x|_{2}, x \in \mathbb{R}^{k}$. Then we have $\frac{1}{\lambda^{k}\left(B_{\left.|\cdot|_{\tau}\right)}\right.} \leq 2^{2 k}(k-1)^{k-1}(\tau+M)^{k-1} \tau$ and therefore

$$
\mathcal{H}^{k,|\cdot|_{\tau}}(A) \leq \tau 2^{2 k}(k-1)^{k-1}(\tau+M)^{k-1} \lambda^{k}(A)
$$

for all $A \subset \mathbb{R}^{k}$.

Proof: We can assume $k>1$ because in the case $k=1$ every seminorm which is not a norm is identically zero.

Take $0 \neq z \in \mathbb{R}^{k}$ such that $\|z\|=0$ and $|z|_{2}=1$. Further take $\left\{z, e_{2}, \cdots, e_{k}\right\}$ an ONB of Euclidean space $\mathbb{R}^{k}$ and $x:=\lambda_{1} z+\sum_{j=2}^{k} \lambda_{j} e_{j}$ with $\left|\lambda_{1}\right| \leq \frac{1}{2 \tau}$ and $\left|\lambda_{j}\right| \leq \frac{1}{2(k-1)(\tau+M)}$ for all $2 \leq j \leq k$. Then we have

$$
\begin{aligned}
|x|_{\tau} & =\|x\|+\tau|x|_{2} \leq \sum_{j=2}^{k}\left|\lambda_{j}\right|\left\|e_{j}\right\|+\tau \sum_{j=1}^{k}\left|\lambda_{j}\right| \leq \tau\left|\lambda_{1}\right|+(\tau+M) \sum_{j=2}^{k}\left|\lambda_{j}\right| \\
& \leq \frac{1}{2}+\frac{1}{2}=1 .
\end{aligned}
$$

Therefore we have $\frac{1}{\lambda^{k}\left(B_{|\cdot|_{\tau}}\right)} \leq 2^{k} \tau(k-1)^{k-1}(\tau+M)^{k-1}$. Because $\mathcal{H}^{k,|\cdot|_{\tau}}=\frac{2^{k}}{\lambda^{k}\left(B_{|\cdot|_{\tau}}\right)} \lambda^{k}$ this proves the lemma.

Now we show that the $\frac{k}{2}$-dimensional Hausdorff measure w.r.t. Kullback-Leibler distance and the $k$-dimensional Hausdorff measure w.r.t. Hellinger distance are proportional to Jeffreys' prior.

\section{Theorem 3.3}

(a) If the regularity conditions $\mathrm{C} 1$ are satisfied we have $\mathcal{H}^{k, d}\left(\Theta^{\prime}\right)=\frac{1}{\omega_{k}} \int_{\Theta^{\prime}} \sqrt{\operatorname{det} I(\vartheta)} d \mu$ for all Borel sets $\Theta^{\prime} \subset \Theta \subset \mathbb{R}^{k}$.

(b) If the regularity conditions $\mathrm{C} 2$ are satisfied we have $\mathcal{H}^{\frac{k}{2}, \mathcal{K}}\left(\Theta^{\prime}\right)=\frac{2^{\frac{k}{2}}}{\omega_{k}} \int_{\Theta^{\prime}} \sqrt{\operatorname{det} I(\vartheta)} d \mu$ for all Borel sets $\Theta^{\prime} \subset \Theta \subset \mathbb{R}^{k}$. 
Proof: The proof of part (a) is an immediate consequence of [8, Theorem 7] because in this case it is $\frac{2^{k}}{\lambda^{k}\left(B_{|\cdot| \vartheta}\right)}=\frac{\sqrt{\operatorname{det} I(\vartheta)}}{\omega_{k}}$.

Now let us prove (b). By assumption the map $\varphi: \Theta \rightarrow L^{1}(\mu), \vartheta \mapsto f_{\vartheta}$ is injective and continuous, hence by Kuratowski's theorem (cf. [10, Cor. 3.3]) Borel sets w.r.t. Euclidean metric are also Borel sets w.r.t. total variation metric. Further, by [7, Theorem 6.11] we have $\left\|f_{\vartheta}-f_{\vartheta^{\prime}}\right\|_{1} \leq \sqrt{2 \mathcal{K}\left(\vartheta, \vartheta^{\prime}\right)}$ for all $\vartheta, \vartheta^{\prime} \in \Theta$. Therefore corollary 2.3 implies that Borel subsets of $\Theta$ are measurable w.r.t. the Hausdorff measure $\mathcal{H}^{\frac{k}{2}}, \mathcal{K}$.

First we consider the parameter set $\Theta_{1}:=\{\vartheta \in \Theta \mid \operatorname{det} I(\vartheta)>0\}$. This is an open subset of $\mathbb{R}^{k}$ and for each $\vartheta \in \Theta|y|_{\vartheta}:=\sqrt{\langle y, I(\vartheta) y\rangle}$ defines a norm on $\mathbb{R}^{k}$. Hence by proposition 3.1 for each $\vartheta \in \Theta_{1}$ and $\varepsilon>0$ there is a $\delta>0$ such that

$$
(1-\varepsilon)\left|\vartheta^{\prime}-\vartheta^{\prime \prime}\right|_{\vartheta} \leq \sqrt{2} \sqrt{K\left(\vartheta^{\prime}, \vartheta^{\prime \prime}\right)} \leq(1+\varepsilon)\left|\vartheta^{\prime}-\vartheta^{\prime \prime}\right|_{\vartheta}
$$

for all $\vartheta^{\prime}, \vartheta^{\prime \prime} \in B(\vartheta, \delta)$. Therefore we get by the area-formula 2.7

$$
\mathcal{H}^{\frac{k}{2}, \mathcal{K}}\left(E \cap \Theta_{1}\right)=\mathcal{H}^{k, \sqrt{\mathcal{K}}}\left(E \cap \Theta_{1}\right)=2^{-\frac{k}{2}} \int \frac{2^{k}}{\lambda^{k}\left(B_{|\cdot| \vartheta}\right)} d \mu=\frac{2^{\frac{k}{2}}}{\omega_{k}} \int \sqrt{\operatorname{det} I(\vartheta)} d \mu .
$$

Now we consider the parameter set $\Theta_{2}:=\{\vartheta \in \Theta \mid \operatorname{det} I(\vartheta)=0\}$. As the complement of $\Theta_{1}$ this is closed in $\Theta$ and therefore $\sigma$-compact. We will show $\mathcal{H}^{\frac{k}{2}}, \mathcal{K}\left(E \cap \Theta_{2}\right)=0$ for every Borel set $E \subset \mathbb{R}^{k}$.

First let $M \subset \Theta_{2}$ be compact. Then by proposition 3.1 for each $\varepsilon>0$ there is a $l \in \mathbb{N}$ and parameters $\vartheta_{1}, \cdots, \vartheta_{l} \in M$ and $\delta_{1}, \cdots, \delta_{l}>0$, such that $M \subset \cup_{i=1}^{l} B\left(\vartheta_{i}, \delta_{i}\right)$ and $\sqrt{2 \mathcal{K}\left(\vartheta^{\prime}, \vartheta^{\prime \prime}\right)} \leq$ $\left|\vartheta^{\prime}-\vartheta^{\prime \prime}\right|_{\vartheta_{i}}+\varepsilon\left|\vartheta^{\prime}-\vartheta^{\prime \prime}\right|_{2}$ for all $\vartheta^{\prime}, \vartheta^{\prime \prime} \in B\left(\vartheta_{i}, \delta_{i}\right)$. Let us define $A_{1}:=M \cap B\left(\vartheta_{1}, \delta_{1}\right)$ and $A_{i}:=M \cap B\left(\vartheta_{i}, \delta_{i}\right) \backslash \bigcup_{j=1}^{i-1} B\left(\vartheta_{j}, \delta_{j}\right)$ for $2 \leq j \leq l$, then by lemma 3.2 we have

$$
\begin{aligned}
\mathcal{H}^{\frac{k}{2}, \mathcal{K}}(E \cap M) & =\mathcal{H}^{\frac{k}{2}, \mathcal{K}}\left(\cup_{i=1}^{l} E \cap A_{i}\right)=\sum_{i=1}^{l} \mathcal{H}^{\frac{k}{2}, \mathcal{K}}\left(E \cap A_{i}\right) \\
& \leq \sum_{i=1}^{l} 2^{2 k}(k-1)^{k-1}\left(\varepsilon+\sup _{\vartheta \in M}\|I(\vartheta)\|\right)^{k-1} \varepsilon \lambda^{k}\left(E \cap A_{i}\right) \\
& =2^{2 k}(k-1)^{k-1}\left(\varepsilon+\sup _{\vartheta \in M}\|I(\vartheta)\|\right)^{k-1} \varepsilon \lambda^{k}(E \cap M) \\
& \rightarrow 0 \quad \text { as } \varepsilon \rightarrow 0 .
\end{aligned}
$$

Because $\Theta_{2}$ is $\sigma$-compact this yields $\mathcal{H}^{\frac{k}{2}, \mathcal{K}}\left(E \cap \Theta_{2}\right)=0$ for all Borel sets $E \subset \mathbb{R}^{k}$ and the theorem is proved.

\section{References}

[1] N. Aronszajn. Differentiability of Lipschitz functions in Banach spaces. Studia Math., 57:147-160, 1976.

[2] H. Bauer. Maß und Integrationstheorie. de Gruyter, Berlin - New York, 1990.

[3] W. Doster. Zur Berechnung des Hausdorffmaßes von Familien von Wahrscheinlichkeitsmaßen. PhD thesis, Universität Kaiserslautern, 1995.

[4] K.J. Falconer. Fractal Geometry. John Wiley \& Sons, West Sussex, 1990.

[5] I.A. Ibragimov and R.Z. Has'minskii. Statistical Estimation - Asymptotic Theory. Springer Verlag, New York Heidelberg Berlin, 1981.

[6] H. Jeffreys. Theory of Probability. Clarendon Pr., Oxford, 3rd edition, 1983.

[7] J.H.B. Kempermann. On the optimum rate of transmitting information. In Probability and Information Theory, pages 126 - 169. Lecture Notes in Mathematics, Springer Verlag, 1967. 
[8] B. Kirchheim. Rectifiable metric spaces: Local structure and regularity of the Hausdorff measure. Proc. Amer. Math. Soc., (121):113 - 123, 1994.

[9] S. Kullback. Information Theory and Statistics. Dover Publications, Dover, 1968. Dover Auflage.

[10] K.R. Parthasarathy. Probability measures on metric spaces. Academic Press, New York, 1967.

[11] R.R Phelps. Gaussian null sets and differentiability of Lipschitz maps on Banach spaces. Pac. J. Math., 77:523-531, 1978.

[12] C.A. Rogers. Hausdorff Measures. Cambridge University Press, 1970.

[13] O.G. Smolyanov and H. v. Weizsäcker. Differential families of measures. Journal of Functional Analysis, 118:454-476, 1993. 